\title{
TIPOS DE DATOS Y ESCALAS DE MEDIDA
}

\author{
JoRge DAGnino S. ${ }^{1}$
}

- Los datos son los valores que toma una variable cuando es medida.

- Los datos pueden ser cualitativos y cuantitativos.

- Los cualitativos pueden corresponder a dos categorías, llamados dicotómicos, o a más de dos categorías.

- Los cuantitativos pueden ser continuos o discretos.

- Las escalas de medición se definen por las operaciones matemáticas que pueden ser usadas en su manipulación. Se distinguen escalas nominales, categóricas, de intervalo y de razón.

En cualquier investigación, de un paciente o grupos de estos, lo que hacemos es efectuar mediciones que ayuden a entender, evaluar y diferenciar las características de personas u objetos o grupos de ellas. Así, se miden variables (peso, edad, presión arterial) y los datos son los valores que toma una variable. Estas mediciones nos permiten llegar a cierta precisión en las características de una variable de acuerdo a su cantidad, grado, capacidad o cualidad. Se habla de la precisión de una medición para referirse al grado de exactitud y usualmente esta significa el número de decimales que se alcanza en la medición. El tipo de datos y las escalas de medida son importantes por la precisión implícita y porque determinan qué métodos de análisis son válidos y sensatos.

\section{TIPOS DE DATOS}

Una primera caracterización distingue entre datos cualitativos y cuantitativos, y estos pueden ser continuos o discretos:
1) Los datos cualitativos: pueden corresponder a dos categorías, denominados dicotómicos, (vivo/muerto, si/no) o a más de dos categorías (fumador, no fumador, ex fumador). Los sujetos son clasificados sin un orden.

2) Los datos cuantitativos, pueden ser:

a)Continuos, aquellos en los que es posible, a lo menos teóricamente, observar un número infinito de valores regularmente espaciados entre dos puntos cualesquiera de su intervalo de medidas. En general corresponden a mediciones (por ejemplo, presión arterial, tiempo, peso, potasemia).

b)Discretos, aquellos que sólo pueden tener un número finito de valores en su intervalo de medidas; en general, corresponden a conteos (por ejemplo, número de hijos, estadio de las enfermedades, género, y también edad medida hasta el último cumpleaños o frecuencia cardiaca).

El límite muchas veces lo pone la sensibilidad o precisión de los instrumentos de medición lo que podría provocar confusión entre lo que es continuo y lo que es discreto. Por ejemplo, la edad es continua, pero la edad medida hasta el último cumpleaños es discreta. En la práctica, si se puede efectuar un elevado número de mediciones en el intervalo de medidas posible y si los intervalos entre las mediciones son uniformes, esas medidas pueden considerarse continuas para su análisis.

\section{ESCALAS DE MEDICIÓN}

Otra aproximación es que los datos pueden definirse también por la escala usada para la medición de las variables, distinguiéndose cuatro de ellas: nominales, ordinales, de intervalo y de razón. Los cuatro niveles de medida determinan qué operaciones son posibles de usar y por consiguiente las

Profesor Titular, División de Anestesiología, Pontificia Universidad Católica de Chile. 
pruebas estadísticas que son permisibles en cada nivel. Todas las escalas tienen algunas propiedades formales que proporcionan definiciones de las características de la escala con mayor exactitud que las que puede asignarse en términos verbales. Estas propiedades pueden expresarse más abstractamente especificando las operaciones aritméticas posibles y las relaciones entre los objetos que han sido clasificados.

1) La escala nominal. Comprende los datos nominales que no tienen un órden: los datos dicotómicos solo pueden tomar dos valores posibles (como vivo o muerto). Es el nivel de medición más débil. Los números u otros símbolos se usan simplemente para clasificar un objeto, persona o característica. Ejemplos: número de ficha clínica, de teléfono, de permiso de circulación, números en las camisetas de un equipo de fútbol. En una escala nominal, la operación consiste en dividir una clase determinada en un conjunto de subclases mutuamente excluyentes. La única relación envuelta es la de equivalencia, simbolizada por el signo $=$, o su ausencia, por el símbolo $\neq$.

En las escalas nominales la única operación admisible es la de transformación "uno a uno" o "simétrica". Esto implica que los símbolos en la escala pueden ser intercambiados siempre que esto sea hecho consistente y completamente. Las únicas estadísticas descriptivas admisibles son aquellas que no cambiarían con una transformación de ese tipo: el modo y las frecuencias. Bajo ciertas condiciones se pueden probar hipótesis relacionadas con la distribución de casos en las categorías usando un chi cuadrado o una prueba exacta de Fisher. La prueba de asociación más común para datos nominales es el coeficiente $\mathrm{C}$ de contingencia.

2) Escala ordinal o de rangos. Comprende los datos ordinales con un mínimo de tres valores posibles y con un número total limitado. Los objetos en una categoría no sólo son diferentes de los objetos en otras categorías de esa misma escala sino que tienen alguna relación con ellos. Relaciones típicas son: mayor, más alto, más difícil, más enfermo, menos grave. Su significado específico depende de la naturaleza de la relación que define esa escala. Ejemplos: escalas de clase socioeconómica, grados de un linfoma, grados de Mallampati, clasificación ASA. Muchas pruebas o escalas de evaluación resultan en puntajes que tienen la fortaleza de rangos: el puntaje del Mini Mental State, la escala de Glasgow o el puntaje APACHE son buenos ejemplos. Aun cuando estos puntajes pueden parecer más precisos que los rangos, generalmente no cumplen con los requisitos de escalas de mayor nivel y deben ser consideradas como ordinales.

La diferencia fundamental entre una escala nominal y la ordinal estriba en que ésta incorpora la relación de "mayor que" $(>)$ o "menor que" $(<)$ a la de equivalencia $(=)$. Cualquier transformación que no altere el orden de las clases es permisible. La mejor descripción de la tendencia central es la mediana ya que ésta no se ve afectada por cambios de puntajes, en tanto no se altere el número de cuentas sobre o bajo ella. Con la escala ordinal es posible usar todo el rango de pruebas estadísticas conocidas como "estadísticas ordinales o de rango" o "no paramétricas". La única presunción de estas pruebas es que los puntajes deben ser sacados de una distribución continua.

Las escalas visuales análogas (EVA) pertenecen estrictamente a este grupo y en rigor debieran ser analizadas con pruebas no paramétricas. Sin embargo, algunos autores han aducido que el error introducido por un análisis paramétrico es de escasa magnitud y que no altera las conclusiones, por lo que bien puede aprovecharse la mayor potencia de las pruebas paramétricas, práctica ampliamente aceptada en la literatura anestesiológica.

3) La escala de intervalo. Comprende los datos continuos o datos discretos que contienen un elevado número de posibles valores. Las distancias entre cualquier par de números de la escala tienen una dimensión conocida y constante por lo que es posible conocer con certeza la magnitud de los intervalos. Se caracteriza por tener una unidad común de medida que asigna un número real a todos los pares de objetos en el conjunto ordenado. Aun cuando el punto cero y la unidad de medida son arbitrarios, la razón entre dos intervalos es independiente de esa unidad y de ese punto.

La medición de temperatura se hace en escala de intervalo. Las escalas Fahrenheit y Celsius tienen cada una su unidad arbitraria, los grados, y también un cero arbitrario. Ambas escalas, sin embargo, tienen la misma cantidad y tipo de información pues están linealmente relacionadas; la medida en una escala puede transformarse a la equivalente en la otra mediante una transformación lineal.

Las operaciones y relaciones de una escala de intervalo son similares a la estructura de la aritmética por lo que pueden utilizarse todas las operaciones. En una escala de intervalo se puede reconocer la equivalencia $(=)$ y el órden $(>)$ y, además, las razones de cada intervalo. Las pruebas paramétricas pueden y deben usarse si se cumplen las otras presunciones puesto que las pruebas no paramétricas restan potencia al análisis al no usar toda la información contenida en una escala de intervalo. 
4) Escala de razón. Además de todos los atributos de una escala de intervalos, ésta tiene en su origen un cero verdadero y en ella la razón entre dos puntos de la escala es independiente de la unidad de medida. La masa, el peso, la distancia, el tiempo se miden en esta escala. Por ejemplo, si medimos el peso de dos objetos en gramos o en onzas, la razón es constante, cosa que no sucede si se mide la temperatura de un cuerpo en grados Celsius o Fahrenheit. Este tipo de escalas sólo se obtienen cuando las siguientes cuatro relaciones son operacionalmente posibles: equivalencia, mayor que, razón conocida entre dos intervalos y razón conocida entre dos valores escalares cualesquiera. Los números asociados con esta escala son verdaderos con un cero también verdadero. Cualquier prueba estadística puede ser usada, incluyendo medidas como la media geométrica y el coeficiente de variación que requieren de un punto cero verdadero.

El orden de las escalas indica la cantidad relativa de información que contiene cada una de las ellas. Las mediciones de un nivel de información concreto pueden transformarse o reescalarse a un nivel inferior, pero no es posible reescalar las variables a un nivel superior al que se midieron realmente. Al reescalar a un nivel inferior se pierde información, hecho que tiende a aumentar el error tipo II, de no encontrar una diferencia cuando en realidad existe, si todo lo demás se mantiene igual. En otras palabras, reescalar a un nivel inferior reduce la potencia estadística por lo que debe evitarse; por ejemplo, reducir la edad a dos o más grupos, transformando una variable continua en una categórica.

\section{REFERENCIAS}

1. Bland M. An Introduction to Medical Statistics. 3rd Ed, Oxford: OUP, 2006.

2. Dawson-Saunders B, Trapp RG. Bioestadística Médica. México
D.F: Manual Moderno, 1993.

3. Dexter F, Chestnut DH. Analysis of statistical tests to compare visual analogue scale measurements among groups. Anesthesiology 1995; 82: 896-902.

4. Forrest M, Andersen B. Ordinal scale and statistics in medical research. BMJ 1986; 292: 537-538.

5. Portney LG, Watkins MP. Foundations of Clinical Research. Applications to practice. 2nd ed. Upper Saddle River: PrenticeHall, 2000.
Correspondencia a:

Dr. Jorge Dagnino S.

jdagnino@med.puc.cl 